\title{
A method for evaluating the heat rejection efficiency in a Lunar power plant consisting of a free-piston Stirling engine (FPSE).
}

\author{
Sergey Smirnov ${ }^{1}$, Mikhail Sinkevich ${ }^{1,2}$, Yuri Antipov ${ }^{1}$ and Hassan Khalife $^{1,2^{*}}$ \\ ${ }^{1}$ Peoples' Friendship University of Russia (RUDN University), 6 Miklukho-Maklaya St, Moscow, \\ 117198, Russian Federation \\ ${ }^{2}$ Joint Institute for High Temperatures, Russian Academy of Sciences, 132 Izhorskaya St, Moscow \\ 125412, Russian Federation.
}

\begin{abstract}
A method for evaluating the heat rejection efficiency in a Lunar power plant consisting of a free-piston Stirling engine FPSE is proposed. The waste heat from the FPSE is absorbed by the refrigerant circulating in a closed pumped loop and then rejected through a radiator into space. The magnitude of the heat flux rejected through the radiator is determined by the temperature difference between the radiator fins and surrounding environment, as well as the surface areas of the radiator and the emissivity coefficient. The method developed is used to qualitatively evaluate the refrigerant efficiency based on calculating the average temperature of the radiator fin which is established during the heat exchange process in the radiator. The method allowed us to determine the most efficient refrigerant in terms of maximum heat rejection at a given operating temperature range without the need of detailed calculations like in the previous works of the authors. Computational studies in a two-dimensional formulation of the radiator, using helium or liquid ammonia as a refrigerant, to determine the quantitative characteristics of the heat rejection process and overall dimensions of the radiator were performed, and a comparative analysis of the results is presented.
\end{abstract}

\section{Introduction}

Humans are closer than ever from building outposts on the surface of the Moon. The future habitants of the moon will require power generation systems to fulfill their energy needs and support their activities, such as scientific experimentation, in situ mining and processing, astronomical observation, and surface exploration. Power generation systems will be located in vacuum, and since waste heat rejection is considered a vital aspect of their nominal operation and convection is not possible for heat rejection, the only mechanism of rejecting heat in space is through radiation. [1]

Due to the extreme low temperatures and harsh surrounding environment in space, the heat rejection process is considered quite difficult and has high requirements regarding the

\footnotetext{
${ }^{*}$ Corresponding author : hassan.khalife.rudn@gmail.com
} 
design. The power generation system considered in this paper is the free-piston Stirling engine FPSE, which has proven its effectiveness in space conditions, and is planned to be used in future space exploration missions.[2] One of the important aspects for the efficient and reliable operation of the FPSE, is maintaining the minimum temperature of the working gas in the engine at a given level. On the moon, the low ambient temperature makes it possible to achieve minimal temperature of the working fluid inside the FPSE, which would improve the overall efficiency of the power generation system. Therefore, designing a highly efficient heat rejection system and investigating capabilities of the FPSE heat rejection system are crucial for the nominal performance and maximum efficiency of the power generation system.[3]

Several heat rejection systems are used nowadays in space, one of which are widely used, the fluid loop system. This system transfers heat from the equipment, the power generation system in our case, to the radiators, which then rejects the heat into free space. This system can be a single-phased loop, controlled by a pump, or a two-phased loop, composed of heat pipes. In this work we consider a single-phase pumped loop heat rejection system, which is a continuation of our previous work [4]. In our previous work, we proposed a method for calculating the radiator temperatures in a one-dimensional and two-dimensional formulation for a lunar power plant. As an example, the calculations were carried out using helium as a refrigerant inside the heat rejection system.

We continued our research in this area and developed a methodology for evaluating the efficiency of the refrigerant for rejecting heat through the radiator of the FPSE's heat rejection system. To check the validity of the method, we compared ammonia in its two states, liquid and gaseous, and gaseous helium.

The choice of ammonia as a refrigerant is due to its proven advantages in space cooling systems. For instance, the International Space Station has ammonia as a refrigerant for key components of the Station's thermal control system. The cooling system on board of the ISS keeps the astronauts comfortable and prevents delicate electronic systems from being frozen or overheated.

Ammonia has several advantages that makes it quite effective in space applications. First, its high thermal capacity. Ammonia stores and transports heat without high pumping power, which gives it a huge advantage when compared to other refrigerants. Second, its wide range of operating temperatures. It can reach very low temperatures and still be pumpable. Its viscosity allows the minimum pumping power through pipes, and its $30 \%$ lighter in weight when compared to water. Last but not least, ammonia is widely available and cheap. [5]

The aim of this paper is to develop a method for evaluating the heat rejection efficiency of a refrigerant in the heat rejection system of an FPSE. The validity of the method is proved by the comparative analysis of the use of helium or ammonia as a refrigerant in the heat rejection system of the FPSE designed to operate in a Lunar power plant.

\section{Overview of the heat rejection system of the FPSE}

The FPSE powered by a nuclear reactor was considered in this work.[6] The overview scheme of the FPSE and the heat rejection system is shown in Fig. 1. 


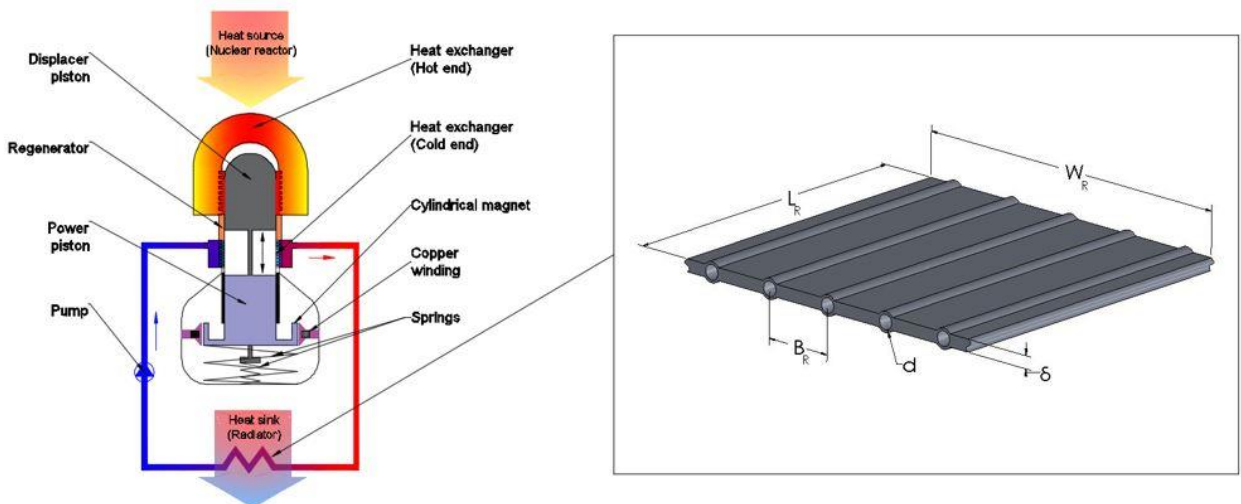

Fig. 1. Overview scheme of the FPSE and the heat rejection system

Heat is supplied from the nuclear reactor to the FPSE through a heat exchanger. Inside the FPSE the working fluid, helium, expands and contracts, passing back and forth through the heat exchangers (regenerator, cold and hot heat exchangers) due to differences in temperature and pressure between the displacer and power pistons. The power piston is connected to a reciprocating cylindrical permanent magnet in a linear alternator, where mechanical energy is transformed into electrical energy and supplied to the consumer. Afterwards heat is rejected from the FPSE through a radiator. Heat from the nuclear reactor can be also utilized to power additional power generation units, however, only one FPSE was considered in the calculations of this work.

In this work, the design of the heat rejection system developed by the authors was used. It consists of two circuits. The first circuit is the circulating working fluid inside the FPSE, while the second circuit is the heat rejection system which consists of a pump and a radiator in which the refrigerant circulates. Structurally, the radiator is presented in the form of heat transport pipes wrapped tightly with aluminum fins. Aluminum is the primary metal used in radiator panels due to its low density and high thermal conductivity. The fins maximize heat conduction from the surface of the pipes to the bulk fin material.

\section{Calculation method}

The heat rejection process starts when the refrigerant exits the FPSE cold heat exchanger and is directed towards the radiator, where heat is transferred from the refrigerant while passing through the radiator pipes to the walls of the pipes, as a result of convective heat transfer $\left(Q_{\text {conv }}\right)$, and then heat is rejected by the radiator fins $\left(Q_{\text {rad }}\right)$ into the surroundings.[7,8] As a result, the following condition expressing the heat exchange must be met:

$$
Q_{\text {rad }}=Q_{\text {conv }}
$$

The amount of heat that must be rejected from the refrigerant through one pipe is calculated as follows:

$$
Q_{l}=\Delta H_{l}^{*}
$$

and 


$$
Q_{l}=Q_{\text {rad }}=Q_{\text {conv }}
$$

In our previous work [4], the dynamic component was not taken into account due to the small difference in velocities at the inlet and outlet of the radiator pipe and the specific enthalpies $h_{l}$ in $\mathrm{J} / \mathrm{kg}$ were considered, then the amount of heat contained in the refrigerant is calculated as follows:

$$
Q_{l}=m_{r e f} \Delta h_{l}
$$

Now, considering the rate of heat transfer in one pipe:

$$
q_{l}=G_{r e f} \Delta h_{l}
$$

The flow rate of the refrigerant must be constant and is determined by the following formula:

$$
G_{r e f}=\rho_{i n} \cdot w_{i n} \cdot \frac{\pi \cdot d^{2}}{4}
$$

The number of radiator pipes required for the heat rejection process:

$$
n_{p}=\frac{q_{0}}{q_{l}}
$$

Where $q_{0}$, is the total heat that should be rejected into space.

The rate of heat transferred as a result of convection is calculated as follows:

$$
q_{c o n v}=\alpha \cdot \pi \cdot d \cdot L_{R} \cdot\left(T-T_{w}\right)
$$

The heat transferred to the walls of the radiator pipes, is then distributed along the fins of the radiator by heat conduction., and afterwards rejected to the surroundings through radiation from the surface of the radiator $q_{R}$ :

$$
q_{R}=\sigma \cdot \varepsilon \cdot 2 \cdot L_{R} \cdot\left(B_{R}+d_{R}\right) \cdot\left(\mathrm{T}_{R}^{4}-\mathrm{T}_{S}^{4}\right)
$$

From the condition of equal heat transfer rates $\left(q_{c o n v}=q_{R}\right)$, the thermal state of the radiator fins was determined.

The method for determining the temperature state in a two-dimensional formulation is described in our work [4]. Computational studies have been carried out using helium as a refrigerant in the heat rejection system of an FPSE.

The amount of heat radiated from the surfaces of the radiator at a constant ambient temperature is determined by the surface area and the temperature level in the radiator. From formula (9) we can deduce that, since the temperatures are in the fourth degree, the greater the difference between the surface temperature $T_{R}$ and the ambient temperature $T_{S}$, the more efficient the heat rejection is. In other words, the heat flux depends on the fin temperature.

Therefore, for a preliminary evaluation of the efficiency of the refrigerant, it is necessary to determine the average temperature of the fin. The equation for determining the average temperature of the fin was obtain using the following condition:

$$
q_{\text {conv }}=q_{R}
$$


The heat flux during convective heat transfer from the refrigerant to the walls of the radiator pipe is determined by the formula (8), hence:

$$
T_{w}=T_{h}-\frac{q_{\text {conv }}}{\alpha \pi d l}
$$

Where the mean temperature of the refrigerant is:

$$
T_{\text {mean }}=\frac{T_{\text {in }}+T_{\text {out }}}{2}
$$

Using formula (7) and assuming $\left(T_{R}=T_{w}\right)$ and the condition of equality of the heat fluxes $\left(q_{\text {conv }}=q_{R}\right)$, we obtain the following:

$$
T_{R}=T_{\text {mean }}-\frac{\sigma \varepsilon 2\left(B_{R}+d_{R}\right) L_{R}\left(T_{R}^{4}-T_{S}^{4}\right)}{\alpha \pi d l}
$$

Where the heat transfer coefficient is equal to

$$
\alpha=N u \frac{\lambda}{d}
$$

After combining the equations, we obtain the design equation for determining the average temperature of the radiator fin:

$$
A T_{R}^{4}+T_{R}+B=0
$$

Where,

$$
\begin{gathered}
A=\frac{2 \sigma \varepsilon B_{R}}{\pi N u \lambda} ; \\
B=-A T_{S}^{4}-T_{\text {mean }}
\end{gathered}
$$

The Nusselt number for turbulent flow was determined as follows:

Where,

$$
N u_{0}=\frac{\left(\frac{f}{8}\right) \cdot R e \cdot P r}{R_{1}+R_{2} \cdot \sqrt{\frac{f}{8}}\left(\operatorname{Pr}^{n}-1\right)}
$$

$$
\begin{gathered}
\operatorname{Pr}=\frac{v}{a} ; \\
R_{1}=1 ; \\
R_{2}=12.7 \\
n=\frac{2}{3} ; \\
f=\left[0.79 \cdot \ln \left(\frac{R e}{8}\right)\right]^{-2}
\end{gathered}
$$

Equation (15) with respect to the unknown temperature $T_{R}$ was solved using the numerical Newton method.

Next, the amount of heat $q_{\text {conv }}$ rejected from the length of a $1 \mathrm{~m}$ pipe and pipe length were determined be $1 \mathrm{~m}$ : 
Where,

$$
L_{R}=\frac{q_{l}}{q_{\text {conv }}}
$$

$$
q_{l}=\frac{q_{s}}{n_{p}}
$$

Then the area of the radiator section and the heat flux rejected from the radiator surface area was determined:

$$
q_{R}=\frac{q_{l}}{L_{R}\left(B_{R}+d_{R}\right)}
$$

The heat transfer coefficient $\alpha$ from the refrigerant to the wall of the cylindrical pipe highly depends on the flow regime of the liquid in the pipe. The studies performed by the authors in previous works have shown that efficient heat transfer occurs during a turbulent flow. The flow regime is determined by the Reynolds number:

$$
\mathrm{Re}=\frac{\mathrm{w} \cdot \mathrm{d}}{\mathrm{v}}
$$

If you set the mean value of the Reynolds number, you can determine the diameter of the radiator pipe and the flow rate of the refrigerant. The necessary formulas are derived below.

Taking into account the above conditions, a formula was derived for determining the pipe diameter $d$ and the refrigerant flow rate at the inlet $w_{i n}$ at a given average Reynolds number $\mathrm{Re}_{\text {mean. }}$. The Reynolds number of the refrigerant flow at the pipe inlet was determined as follows:

and

$$
R e_{\text {in }}=\frac{2 \cdot R e_{\text {mean }}}{\frac{\mu_{\text {in }}}{\mu_{\text {out }}}+1}
$$

Rearranging the formula:

$$
R e_{i n}=\frac{w_{i n} \cdot d}{v_{i n}}
$$

$$
w_{\text {in }} \cdot d=R e_{i n} \cdot v_{i n}
$$

Representing the total mass flow rate of the refrigerant:

$$
G_{T}=\rho_{\text {in }} \cdot \frac{\pi}{4} \cdot n_{p} \cdot w_{\text {in }} \cdot d \cdot d
$$

And combining (21) with (23):

$$
G_{T}=\rho_{\text {in }} \cdot \frac{\pi}{4} \cdot n_{p} \cdot R e_{i n} \cdot v_{i n} \cdot d
$$

Rearranging (24) we get the pipe diameter:

where,

$$
d=\frac{G_{T}}{\frac{\pi}{4} \cdot n_{p} \cdot R e_{i n} \cdot \mu_{i n}}
$$

$$
\mu_{\text {in }}=\rho_{\text {in }} \cdot v_{\text {in }}
$$

The total flow rate $\mathrm{G}_{\mathrm{T}}$ is determined by the total heat flow $\mathrm{q}_{\mathrm{s}}$, which must be rejected and by the temperatures of the refrigerant at the inlet and outlet of the radiator: 
Where,

$$
G_{T}=\frac{q_{s}}{\Delta h_{l}}
$$

$$
\Delta h_{l}=h_{\text {in }}-h_{\text {out }}
$$

Thus, by setting the total flow rate $\mathrm{G}_{\mathrm{T}}$, the number of radiator pipes $\mathrm{n}_{\mathrm{p}}$, the inlet and outlet parameters of the dynamic viscosity of the refrigerant at $T_{\text {in }}$ and $T_{\text {out }}$, the average Reynolds number of the refrigerant flow $\mathrm{Re}_{\text {mean }}$, we were able to determine the pipe diameter $d$ and then the refrigerant flow velocity at pipe inlet $w_{i n}$ :

$$
w_{i n}=\frac{R e_{i n} \cdot v_{i n}}{d}
$$

Hydraulic losses inside the pipes play an important role in the operation of the radiator. The power spent on pumping the refrigerant through the radiator is calculated as follows:

$$
N_{R}=\frac{G_{T} \cdot \Delta P_{R}}{\eta \cdot \rho_{\text {mean }}}
$$

Where,

$$
\Delta P_{R}=f \cdot \frac{L_{R}}{d} \cdot \frac{\rho_{h} \cdot w_{h}^{2}}{2}
$$

\section{Results and discussion}

The initial data for the radiator calculation method are:

1. The parameters of the FPSE;

2. The ambient temperature on the surface of the moon;

3. The thermodynamic properties of the refrigerant used in the heat rejection system.

The FPSE model considered in this work, is the same on considered in our previous work, and is one of the models that NASA is planning to use in their KRUSTY project.[9] The main parameters of the FPSE are shown in Table 1.

Table 1. The main parameters of the FPSE.

\begin{tabular}{ccc}
\hline Parameter & Value & Unit \\
\hline Output Power & 1100 & W \\
Total thermal power & 3600 & W \\
Heat to be rejected & 2500 & W \\
Efficiency & 30 & $\%$ \\
Maximum cycle temperature & 600 & $\mathrm{C}$ \\
Minimum cycle temperature & 50 & $\mathrm{C}$ \\
\hline
\end{tabular}

The ambient temperature in this study is of great importance, due to its direct impact on the effectiveness of the heat rejection process. Temperatures on the Moon are extreme, 
ranging from boiling hot to freezing cold depending on where the sun is shining. [10] Ranges of temperature changes relative to their location on the Moon are shown in Table 2.[11]

Table 2. Ranges of temperature changes relative to their location on the Moon

\begin{tabular}{ccc}
\hline Location & Maximum temperature, $\mathrm{K}$ & Minimum temperature, $\mathrm{K}$ \\
\hline Equator & 400 & 100 \\
North pole & 200 & 50 \\
South pole & 200 & 50 \\
Craters & 30 & 20 \\
\hline
\end{tabular}

The location of the lunar power plant assumed in this work was at one of the poles, where the maximum ambient temperature reaches $200 \mathrm{~K}$ (-73 C). At lower ambient temperatures, the difference between the fin temperature and ambient temperature increases, consequently, leading to an increase in the heat rejection efficiency.

Helium and ammonia were considered as the refrigerants circulating inside the heat rejection system of the FPSE, their main properties (gas constant, enthalpy, heat capacity Cp, dynamic viscosity, thermal conductivity) were set according to the data taken from [12].

To compare the efficiency of the refrigerants, the following conditions were set constant:

1. Heat, which must be rejected from the FPSE model considered in this work through the radiator. $\left(\mathrm{q}_{0}=2500 \mathrm{~W}\right)$;

2. Inlet and outlet refrigerant temperatures of the radiator pipe. Three temperature ranges $\Delta \mathrm{T}=\mathrm{T}_{\text {in }}-\mathrm{T}_{\text {out }}$ were considered:

a. $\Delta \mathrm{T}=300-280\left(\mathrm{~T}_{\text {mean }}=290 \mathrm{~K}\right)$;

b. $\Delta \mathrm{T}=280-260\left(\mathrm{~T}_{\text {mean }}=270 \mathrm{~K}\right)$;

c. $\Delta \mathrm{T}=260-240\left(\mathrm{~T}_{\text {mean }}=250 \mathrm{~K}\right)$.

3. The refrigerant flow regime inside of the radiator pipes is turbulent. $\left(R e_{\text {mean }}=\right.$ 4400)

To evaluate the efficiency of the refrigerant used, the heat fluxes emitted from the surfaces of the radiator $\mathrm{q}_{\mathrm{R}}$ and the power $\mathrm{N}_{\mathrm{R}}$ spent on pumping the refrigerant through the radiator were compared.

The temperature ranges were chosen as follows. The first range $(300 / 280)$ was chosen considering the minimum temperature of the working gas of the considered FPSE $(323 \mathrm{~K})$, usually a drop of $20-30 \mathrm{~K}$ is necessary for efficient heat rejection from the working gas to the refrigerant in the FPSE cold heat exchanger. The range $(260 / 240)$, is based on the considered ambient temperature $(200 \mathrm{~K})$, a further decrease in temperature is impractical, since the temperature of the fin will approach the ambient temperature, which will lead to a significant deterioration in the heat transfer process.

Previous studies performed by the authors have shown that the heat transfer coefficient $\alpha$ from the refrigerant to the wall of the pipes in a turbulent flow is much higher when compared to laminar flow. However, as the flow rate increases, the resistance increases and therefore more pumping power is needed to circulate the refrigerant through the radiator. Therefore, the average Reynaud's number $R e_{\text {mean }}=4400$ was set close to the minimum values of pumping power during the turbulent flow regime.

The refrigerant pressures were considered in the range of $0.1-3.0 \mathrm{MPa}$, which made it possible to investigate ammonia in the above temperature ranges in both its gaseous and liquid states (Fig. 2). 


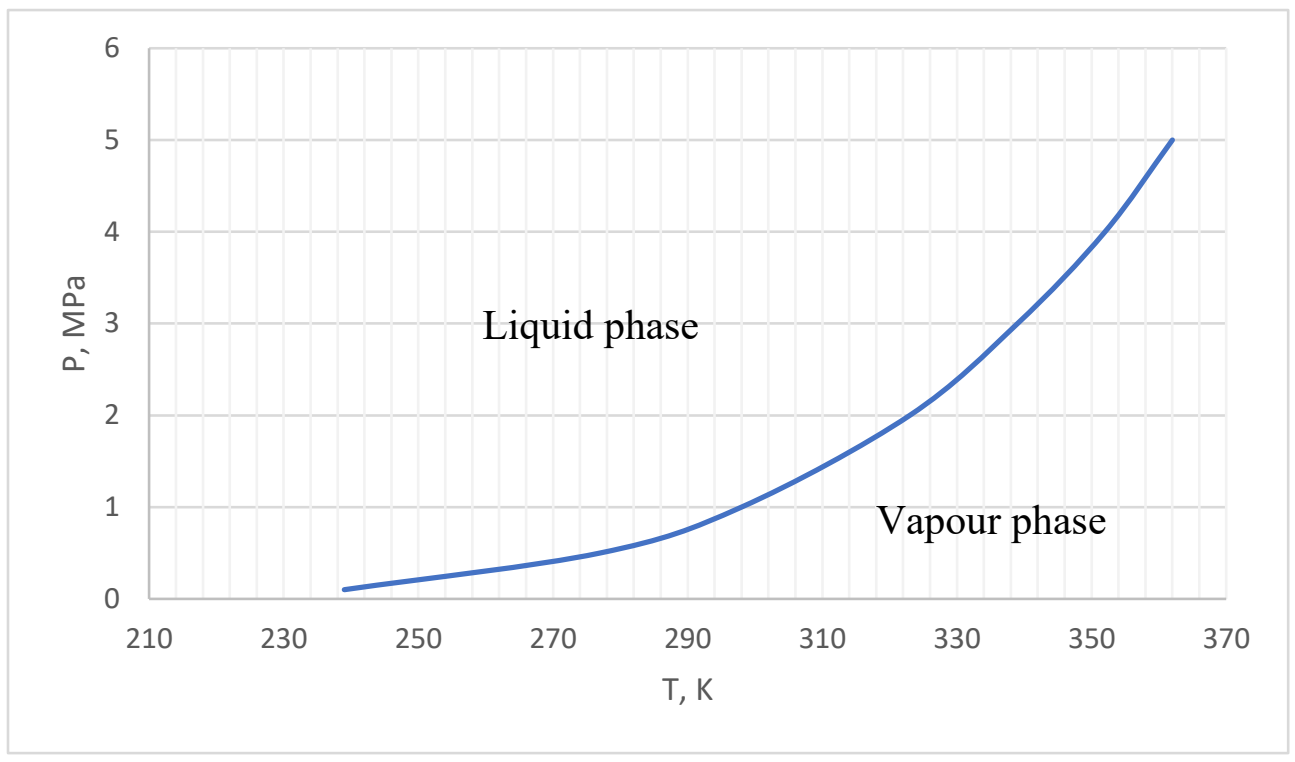

Fig.2. Phase diagram of ammonia

An analysis of formula (8) shows that when setting a constant rejected heat, the greater the heat transfer coefficient, the smaller the temperature difference between the refrigerant $T_{h}$ and the pipe wall $T_{w}$ and, therefore, according to formula (9) the higher the temperature $\mathrm{T}_{\mathrm{w}}$, the higher amount of heat flux will be emitted by the radiator.

Initially, the efficiency of using gaseous ammonia or helium as a refrigerant was compared. The results shown in Fig.3 shows that helium is much more efficient during heat rejection when compared to gaseous ammonia. With the transition of ammonia into the liquid state, the efficiency of heat rejection increases sharply, which is explained by a change in its thermophysical properties. The thermal conductivity coefficient, density and dynamic viscosity of liquid ammonia significantly exceed those of helium. For instance, for $\mathrm{P}=1$ $\mathrm{MPa}, \Delta \mathrm{T}=280-260$, the heat transfer coefficient when using helium is $109 \mathrm{~W} /\left(\mathrm{m}^{2} . \mathrm{K}\right)$, while for liquid ammonia it was $488 \mathrm{~W} /\left(\mathrm{m}^{2}\right.$. K). The heat emitted by the radiator $\mathrm{q}_{\mathrm{R}}$ comparisons are shown in Fig.4 Considering that for the same given average Reynolds number, the flow velocity of helium $(1.82 \mathrm{~m} / \mathrm{s})$ is significantly higher than the flow velocity of liquid ammonia $(0.41 \mathrm{~m} / \mathrm{s})$, the pumping power $\mathrm{N}_{\mathrm{R}}$ for helium is higher. 


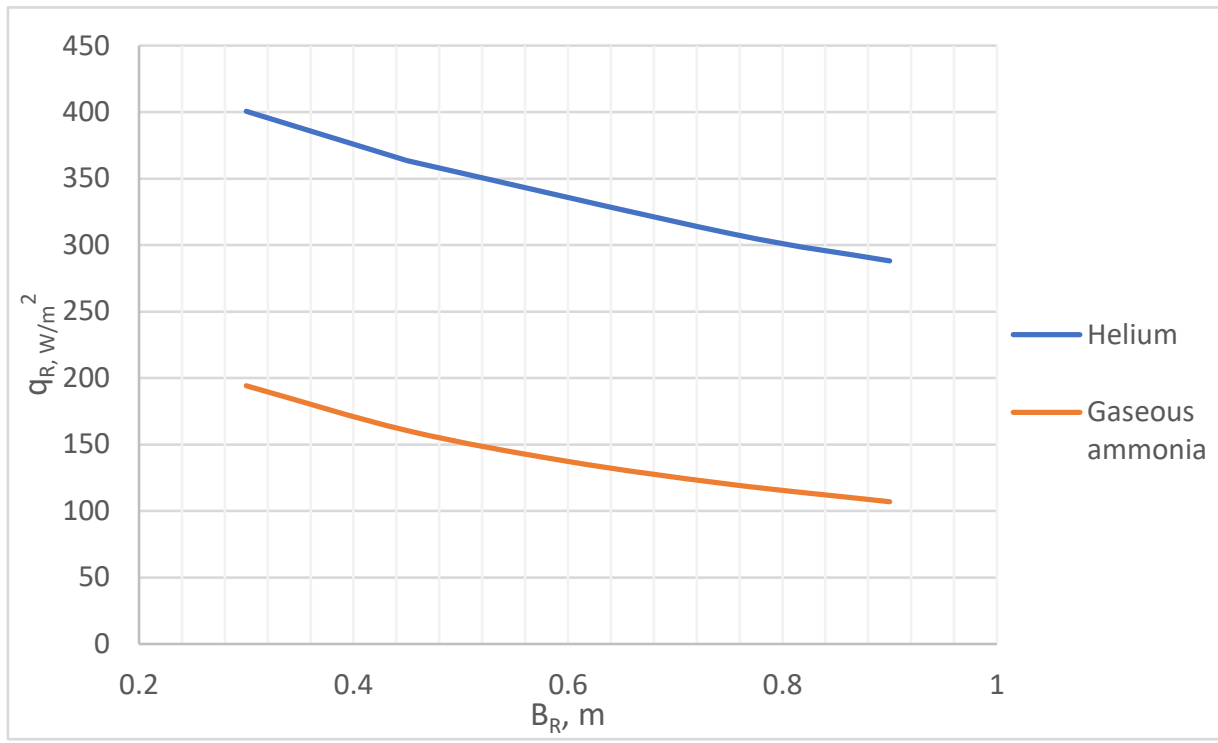

Fig.3. Comparison of helium and ammonia in their gaseous state at $\mathrm{P}=0.5 \mathrm{MPa} \Delta \mathrm{T}=$ $300-280 \mathrm{~K}$

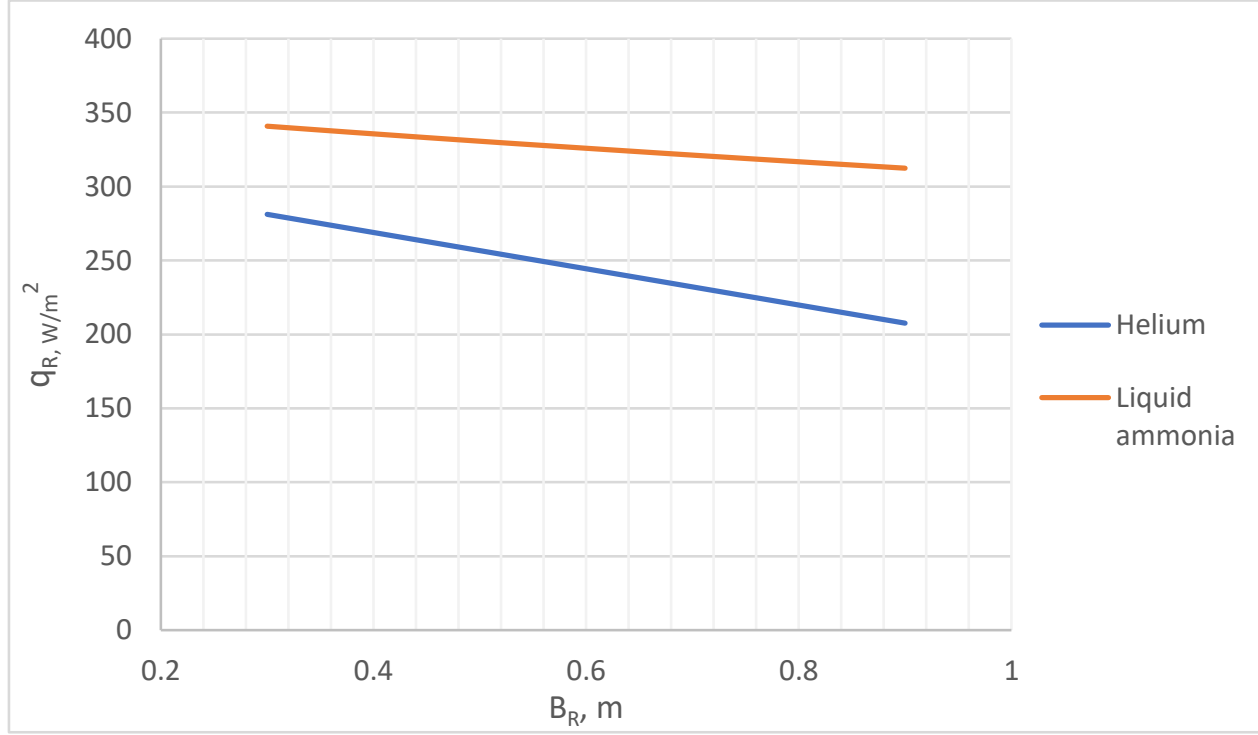

Fig.4. Comparison of gaseous helium and liquid ammonia at $\mathrm{P}=0.5 \mathrm{MPa} \Delta \mathrm{T}=280-260$

\section{$\mathrm{K}$}

An increase in pressure for liquid ammonia has practically no effect on convective heat transfer between the refrigerant and the walls of the radiator pipes, and, consequently, on the heat fluxes emitted from the surfaces of the radiator $\mathrm{q}_{\mathrm{R}}$ and the pumping power $\mathrm{N}_{\mathrm{R}}$, since the changes in thermophysical properties in the considered pressure range $(0.1-3 \mathrm{MPa})$ are insignificant. For helium, which is in a gaseous state, as pressure increases, the density proportionally increases, which makes it possible to proportionally reduce the gas flow rate in the radiator pipe and, therefore, the pumping power $\mathrm{N}_{\mathrm{R}}$ at pressures above $3 \mathrm{MPa}$, becomes less than that of ammonia. The dynamic viscosity and thermal conductivity of helium practically do not change with increasing pressure. When the flow regime is set constant, i.e., 
the Reynolds number, the heat transfer coefficient also changes slightly and, therefore, the heat flux $\mathrm{q}_{\mathrm{R}}$ does not change.

In order to determine the validity of the proposed method in this work (method \#1), the radiator temperatures were calculated in a two-dimensional formulation using the method from our previous work (method \#2), to compare the results. The refrigerant pressure was assumed to be $\mathrm{P}=1.0 \mathrm{MPa}$ and inlet and outlet temperatures of the refrigerant $\Delta \mathrm{T}=280$ 260. The distribution of temperatures on the surface of a fin of the width $B_{R}$, determined by method \# 2, using liquid ammonia or helium, are shown in Fig.5. and Fig.6, respectively.

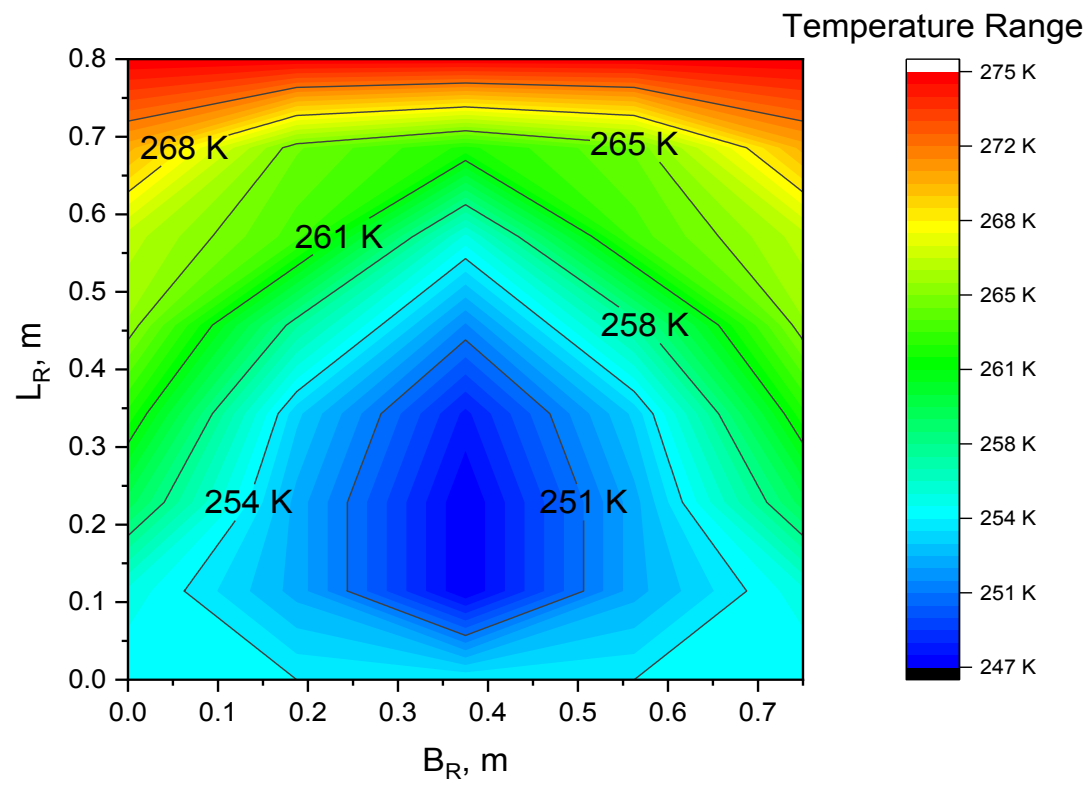

Fig. 5. Temperature distribution on the surface of the radiator fin when using liquid ammonia as a refrigerant 


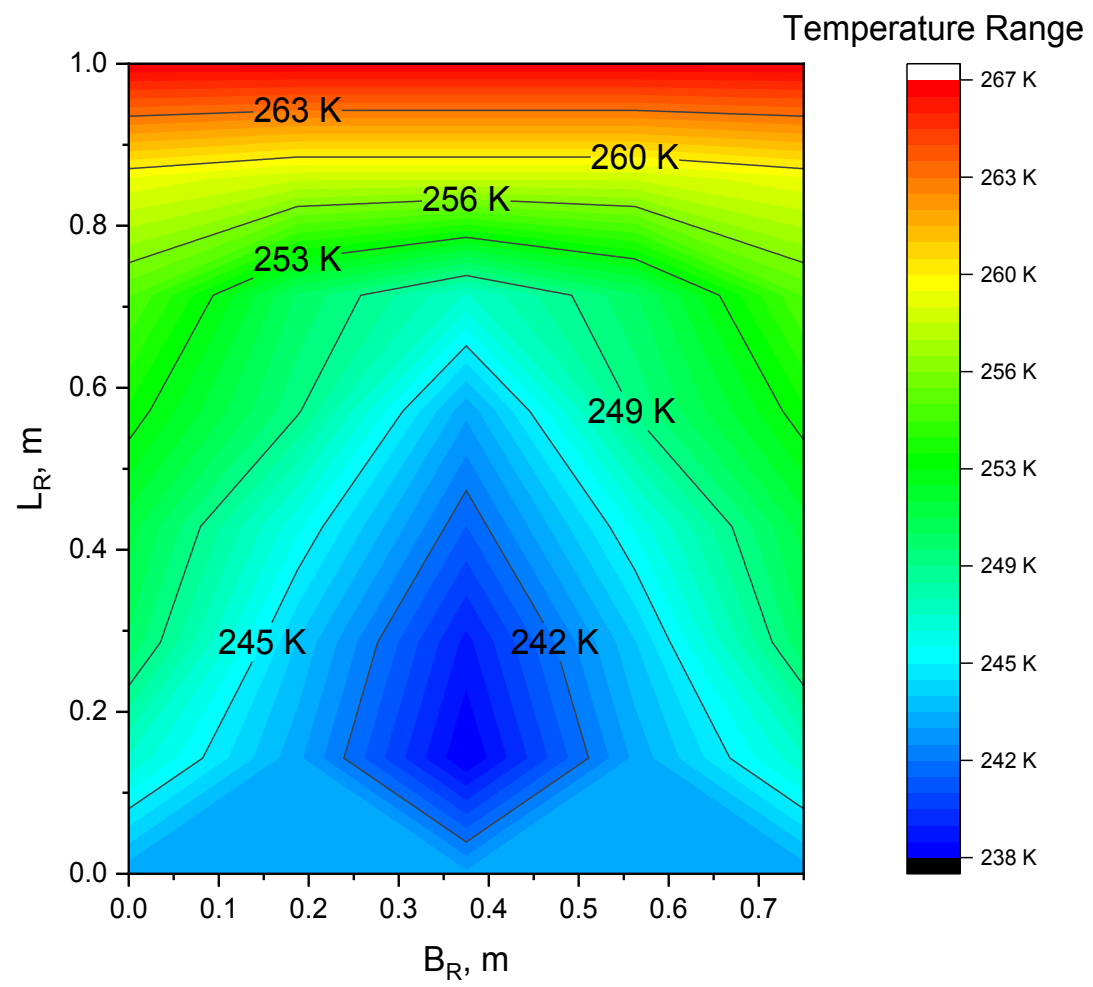

Fig. 6. Temperature distribution on the surface of the radiator fin when using helium as a refrigerant

It is clearly seen that when liquid ammonia is used, the temperature level of the radiator fins is higher and, therefore, the heat flux radiated from the surface of the radiator is higher. The results of the comparison of heat fluxes emitted from the surfaces of the radiator according to methods \# 1 and \# 2 when using liquid ammonia and helium as a refrigerant are shown in Fig. 7 below.

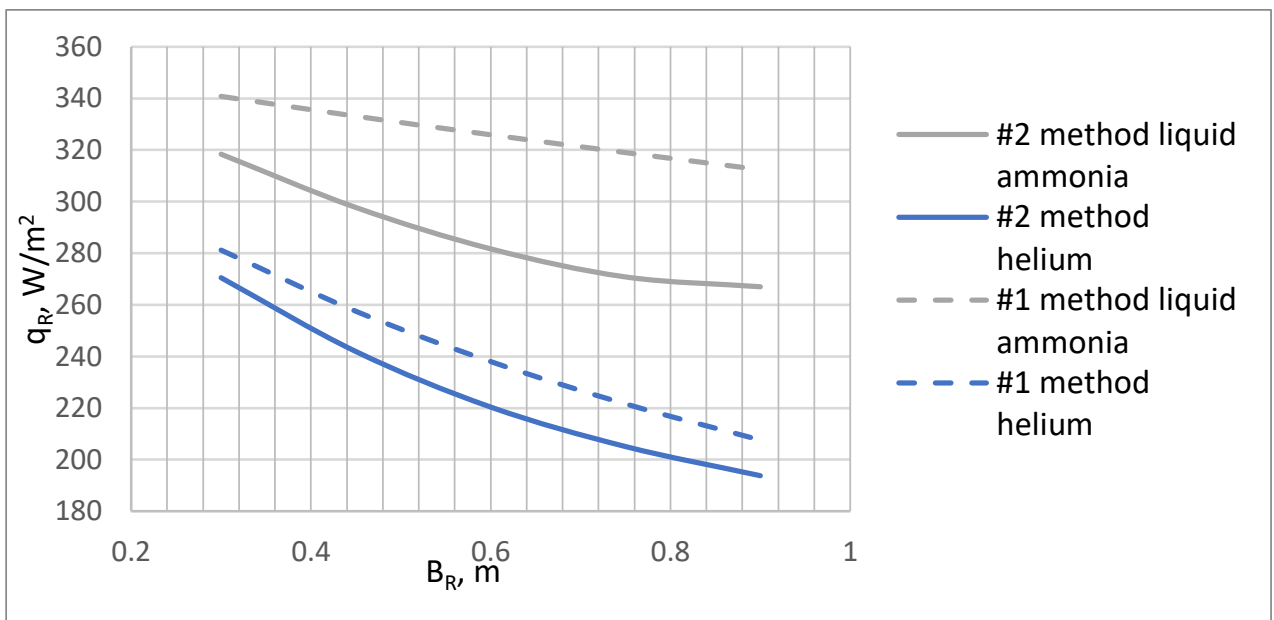

Fig. 7. Comparison of heat fluxes emitted from the surfaces of the radiator according to methods \# 1 and \# 2 
The comparative analysis showed that the developed method in this work for preliminary evaluation makes it possible to qualitatively evaluate the heat rejection efficiency of the refrigerant. Differences in the calculation results using the developed method in this work and the more detailed method developed in our previous work were $7.2-17.8 \%$ for liquid ammonia, and $3.9-8.2 \%$ for helium.

The relatively large error in the study of ammonia is explained by the large difference in thermophysical characteristics with respect to the change in temperature along the length and width of the fin. This is seen due to the averaging of the parameters.

The smallest differences were obtained for narrow fins, due to the smallest temperature unevenness along the width of the fin, therefore, for these fins, it is possible to quantitatively estimate the heat fluxes of radiation. With an increase in the fin width, the method for calculating temperatures in a two-dimensional formulation (\# 2 method) makes it possible to consider the effect of temperature distribution along the length and width of the fin as seen in Fig.5. and Fig.6.

In the fins of a smaller width $B_{R}$, the heat transfer efficiency is higher, since the temperature drop across the fin width $B_{R}$ is less, but at the same time the pipe length increases, which leads to an increase in the hydraulic resistance.

With an increase in the fin width $B_{R}$, the length of the pipe decreases (Fig.8). The temperature of the pipe wall decreases, the uneven distribution of temperatures along the fin width increases, which leads to a decrease in the heat flux rejected.

When using liquid ammonia, the heat rejection efficiency for the considered conditions increases with a fin width of $B_{R}=0.3 \mathrm{~m}$ by $17.7 \%$, and with a fin width of $B_{R}=0.9 \mathrm{~m}$ by $37.8 \%$. The pressure drops in the radiator pipes during the flow mode corresponding to Reynolds numbers in the range 4000 - 5000 are minimal.

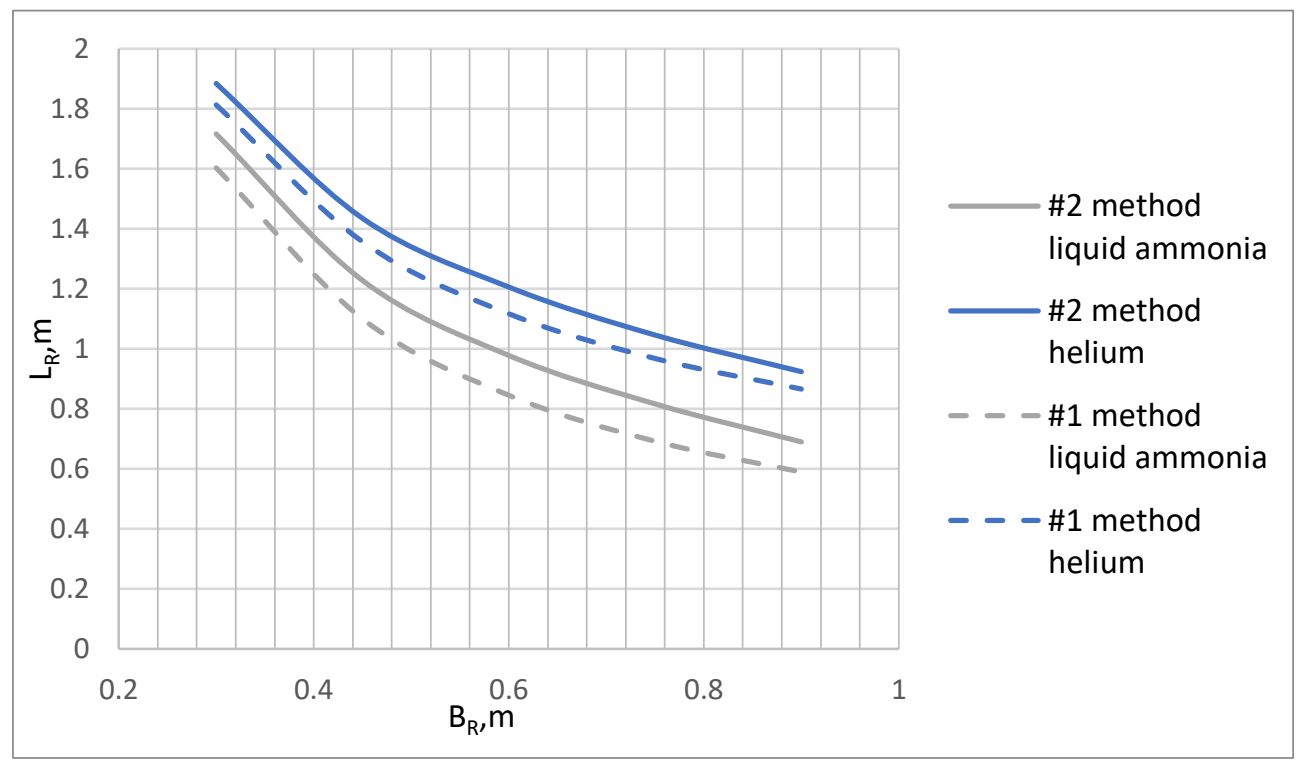

Fig. 8. Comparison of the change in the length of radiator with respect to the fin width according to methods \# 1 and \# 2 
After a preliminary evaluation, choosing a refrigerant for the heat rejection system, then a study is carried out in a two-dimensional formulation of the radiator and the dimensions of the radiator and the pumping power needed for the refrigerant are determined.

The mass of the radiator is determined by the mass of the fins and, with constant fins thickness, by the area of the radiated surface. Therefore, the final choice should be determined after calculating the pressure losses of the entire heat rejection system and possible restrictions on the dimensions of the structure.

A decrease in the temperatures of the refrigerant circulating in the heat rejection system of the FPSE can be achieved by several methods, such as: the development of a more compact cold heat exchanger when cooling the working gas in the FPSE or with the joint modernization of the regenerator and the cold heat exchanger to achieve lower minimum temperatures of the working gas, which will increase the efficiency of the FPSE.

In conclusion, it should be noted that during the transition of liquid ammonia to the gaseous state, its thermophysical characteristics change significantly, therefore, it is necessary to provide measures to exclude changes in the thermal regime and changes in pressure in the heat rejection system of the Stirling engine.

\title{
5 Conclusions
}

Heat rejection systems in space are considered a highly important aspect while developing infrastructure on the moon. This work presents a developed method for evaluating the efficiency of a refrigerant in the heat rejection system in a Lunar power plant consisting of a am FPSE. The major highlights and conclusions of this work are summarized as follows:

1. The validity of the method was proved by the comparative analysis of the use of helium or ammonia as a refrigerant in the heat rejection system of the FPSE designed to operate in a Lunar power plant in a wide range of refrigerant temperatures $(240-300 \mathrm{~K})$ and pressures $(0.1-3,0 \mathrm{MPa})$. Comparative analysis showed that the developed methodology for preliminary evaluation made it possible to qualitatively assess the efficiency of the refrigerant for heat rejection and establish which refrigerant is more efficient for heat rejection. Differences in the calculation results of heat fluxes using the developed method in this work and the more detailed method developed in our previous work were $7.2-17.8 \%$ for liquid ammonia, and $3.9-8.2 \%$ for helium.

2. Studies using the method of calculating the temperatures of the radiator fin in a two-dimensional formulation showed that, when using liquid ammonia, the heat rejection efficiency for the considered conditions increase with a fin width of $\mathrm{B}_{\mathrm{R}}=$ $0.3 \mathrm{~m}$ by $17.7 \%$, and with a fin width of $\mathrm{B}_{\mathrm{R}}=0.9 \mathrm{~m}$ by $37.8 \%$. The pressure drops in the radiator pipes during the flow mode corresponding to Reynolds numbers in the range $4000-5000$ are minimal.

This paper has been supported by the RUDN University Strategic Academic Leadership Program.

\section{Nomenclature}

\author{
$Q_{\text {rad }} \quad$ Amount of heat released due to radiation, J \\ $Q_{\text {conv }} \quad$ Amount of heat released due to convection, J
}


$Q_{l} \quad$ Amount of heat that must be rejected from the refrigerant through one pipe, $\mathrm{J}$

$\Delta H_{l}^{*} \quad$ Change in stagnation enthalpy through one pipe, $\mathrm{J}$

$m_{\text {ref }} \quad$ Refrigerant mass, $\mathrm{kg}$

$\Delta h_{l} \quad$ Change in specific enthalpy through one pipe, $\mathrm{J} / \mathrm{kg}$

$q_{l} \quad$ Rate of heat transferred through one pipe, $\mathrm{W} / \mathrm{m}^{2}$

$G_{\text {ref }} \quad$ Refrigerant mass flow rate, $\mathrm{kg} / \mathrm{s}$

$\rho_{\text {in }} \quad$ Refrigerant density at the pipe inlet, $\frac{\mathrm{kg}}{\mathrm{m}^{3}}$

$w_{\text {in }} \quad$ Refrigerant flow speed at the pipe inlet, $\mathrm{m} / \mathrm{s}$

$d \quad$ The inner diameter of the radiator pipe, $\mathrm{m}$

$n_{p} \quad$ The number of radiator pipes required for the heat rejection process

$q_{0} \quad$ The total rate of heat transfer that should be rejected through the radiator, $\mathrm{W}$

$q_{\text {conv }} \quad$ Rate of heat transferred as a result of convection, $\mathrm{W}$

$\alpha \quad$ Heat transfer coefficient, $\frac{W}{m^{2} \cdot K}$

$L_{R} \quad$ Pipe length, $\mathrm{m}$

T Refrigerant temperature, $\mathrm{K}$

$T_{w} \quad$ Pipe wall temperature, $\mathrm{K}$

$\mathrm{Nu} \quad$ Nusselt number

$\lambda \quad$ Refrigerant thermal conductivity, $\frac{W}{m \cdot K}$

Re Reynolds number

$v \quad$ Refrigerant kinematic viscosity, $\frac{m^{2}}{s}$

Pe Peclet number

Pr Prandtl Number

$q_{R} \quad$ Heat flux transferred as a result of radiation, $\mathrm{W}$

$\sigma \quad$ Stefan-Boltzmann constant, $\frac{W}{m^{2} \cdot K^{4}}$

$\varepsilon \quad$ Emissivity coefficient 
$T_{S} \quad$ Surrounding environment temperature, $\mathrm{K}$

$\lambda_{R} \quad$ Radiator thermal conductivity, $\frac{W}{m \cdot K}$

$T_{R} \quad$ Radiator temperature, $\mathrm{K}$

$\delta \quad$ Fin depth, $\mathrm{m}$

$B_{R} \quad$ Fin width, $\mathrm{m}$

$C_{p} \quad$ Refrigerant specific heat capacity at a constant pressure, $\frac{\mathrm{J}}{\mathrm{kg} \cdot \mathrm{K}}$

$N_{R} \quad$ The power spent on pumping the refrigerant through the radiator, $\mathrm{W}$

$G_{T} \quad$ The total mass flow rate of the refrigerant, $\mathrm{kg} / \mathrm{s}$

$\Delta P_{R} \quad$ Pressure drop due to friction in the radiator pipes, $\mathrm{Pa}$

$\rho_{\text {mean }} \quad$ Refrigerant mean density in the radiator, $\frac{\mathrm{kg}}{\mathrm{m}^{3}}$

$\eta \quad$ Efficiency of the refrigerant pump

$\xi \quad$ Darcy friction factor

$T_{\text {in }} \quad$ Inlet refrigerant temperature, $\mathrm{K}$

$T_{\text {out }} \quad$ Outlet refrigerant temperature, $\mathrm{K}$

$\mu_{\text {in }} \quad$ Refrigerant dynamic viscosity, Pa.s

Subscripts

$\begin{array}{cl}X_{\text {conv }} & \text { Convection } \\ X_{\text {rad }} & \text { Radiation } \\ X_{l} & \text { Relative to one pipe } \\ X_{\text {in }} & \text { Inlet } \\ X_{\text {out }} & \text { Outlet } \\ X_{w} & \text { Pipe wall } \\ X_{\text {ref }} & \text { Refrigerant } \\ X_{R} & \text { Radiator } \\ X_{T} & \text { Total value }\end{array}$




$\begin{array}{cl}X_{p} & \text { Pipe } \\ X_{S} & \text { Surrounding environment } \\ X_{\text {mean }} & \text { Mean value }\end{array}$

\section{References}

1. Hertzberg, Thermal Management In Space.

https://space.nss.org/settlement/nasa/spaceresvol2/thermalmanagement.html (accessed January 21, 2021).

2. M. A. Gibson, S. R. Oleson, D. I. Poston, and P. McClure, in IEEE Aerosp. Conf. Proc. (IEEE Computer Society, 2017), pp. 1-14

3. A. Zajacs, A. Lalovs, A. Borodinecs, and R. Bogdanovics, in Energy Procedia (Elsevier Ltd, 2017), pp. 74-79

4. S. V. Smirnov, M. V. Sinkevich, Y. A. Antipov, and H. S. Khalife, Acta Astronaut. 180, 46 (2021)

5. B. Badger, Ammonia: The Refrigerant of the Future. https://www.energylearning.com/index.php/opinion/112-ammonia-the-refrigerant-of-the-future (accessed January 21, 2021).

6. Using the Sterling Engine for Solar and Lunar Power. https://www.powermag.com/using-the-sterling-engine-for-solar-and-lunar-power/ (accessed March 19, 2021).

7. F.F. Tsvetkov, Problem Book in Heat and Mass Transfer (Publishing house MEI, Moscow, 2008)

8. D. R. .E. Hesselgreaves, R. Law, Compact Heat Exchangers (ButterworthHeinemann, 2016)

9. Kilopower|NASA, (n.d.). https://www.nasa.gov/directorates/spacetech/kilopower (accessed June 12, 2021).

10. What is the Temperature on the Moon? https://www.space.com/18175-moontemperature.html (accessed June 12, 2021).

11. J. P. Williams, D. A. Paige, B. T. Greenhagen, and E. Sefton-Nash, Icarus 283, 300 (2017)

12. Thermophysical Properties of Fluid Systems. https://webbook.nist.gov/chemistry/fluid/ (accessed June 12, 2021). 